\title{
AUTOMORPHISMS OF A SURFACE OF GENERAL TYPE ACTING TRIVIALLY IN COHOMOLOGY
}

\author{
JIN-XING CAI
}

(Received November 19, 2002, revised May 13, 2003)

\begin{abstract}
It is proved that, for a complex minimal smooth projective surface $S$ of general type, any automorphism group of $S$, inducing trivial actions on the second rational cohomology of $S$, is isomorphic to a cyclic group of order less than five or the product of two groups of order two, provided that the Euler characteristic of the structure sheaf of $S$ is larger than 188 .
\end{abstract}

Introduction. It is well-known that, for a curve $C$ of genus $g \geq 2$, the automorphism group Aut $C$ acts faithfully on $H^{1}(C, Q)$.

The case of surfaces has been studied by many authors. For K3 and Enriques surfaces $S$, Aut $S$ acts faithfully on $H^{2}(S, \boldsymbol{Z})$ (cf. [BR], [Ue]); and there exists an Enriques surface $S$ for which Aut $S$ does not act faithfully on $H^{2}(S, \boldsymbol{Q})$ (cf. [Pe]). For compact Kähler surfaces $S$ with $h^{0}\left(T_{S}\right)=0$ and the canonical linear system $\left|K_{S}\right|$ base point free, Peters [Pe] proved that, if a non-trivial $\sigma \in$ Aut $S$ acts trivially on $H^{2}(S, \boldsymbol{Q})$, then either $K_{S}^{2}=8 \chi\left(\mathcal{O}_{S}\right)$ and the order o $(\sigma)$ of $\sigma$ is a power of 2 or $K_{S}^{2}=9 \chi\left(\mathcal{O}_{S}\right)$ and o $(\sigma)$ is a power of 3 .

Taking the product of two hyperelliptic curves, one gets easily examples of surfaces of general type for which Aut $S$ does not act faithfully on $H^{2}(S, \boldsymbol{Q})$. The aim of this paper is to prove the following

THEOREM A. Let $S$ be a complex minimal smooth projective surface of general type, and $\chi\left(\mathcal{O}_{S}\right)$ the Euler characteristic of the structure sheaf of $S$. Let $G \subset$ Aut $S$ be a subgroup of automorphisms acting trivially on $H^{2}(S, \boldsymbol{Q})$. If $\chi\left(\mathcal{O}_{S}\right)>188$, then $G$ is isomorphic to $C_{n}$ $(n \leq 4)$ or $C_{2} \times C_{2}$, where $C_{n}$ is a cyclic group of order $n$.

Theorem A is proved in Sections 2 through 4. Thanks to Beauville's theorem on the canonical map of $S$, the problem reduces to the analysis of the automorphisms of the canonical fiber surface $f: S \rightarrow B$, of genus $g \leq 5$. The main part of this paper is to treat the case $g=3$ and $G$ nonabelian of order 8 or 6 . The idea of the proof is to prove the existence of a $G$ invariant irreducible curve (in a singular fiber of $f$ ) on which $G$ acts faithfully and to analyze the action around it.

2000 Mathematics Subject Classification. Primary 14J50; Secondary 14J29.

This work was supported by the Chinese Natural Science Foundation (No. 10271005) and the Scientific Research Foundation for Returned Overseas Chinese Scholars, the State Education Ministry. 
We use standard notation as in [BPV] or [Ha]. In this paper we denote by $C_{n}, D_{2 n}$ and $Q_{8}$ the cyclic group of order $n$, the dihedral group of order $2 n$, and the quaternion group of order 8.

I am grateful to the referee for a very careful reading of the paper and several valuable suggestions.

1. Preliminaries. For the reader's convenience, in this section we recall several results from the literature.

(1.1) Let $S$ be a smooth complex projective surface of general type, with a fibration $f: S \rightarrow B$ of genus $g \geq 2$ over a smooth curve $B$. We assume that $f$ is relatively minimal, that is, $S$ has no $(-1)$-curves contained in a fiber of $f$. Denote by $F$ the general fiber of $f$. Let $K_{S}$ be the canonical divisor of $S$.

We say that $f$ is a hyperelliptic (resp. nonhyperelliptic) fibration if $F$ is a hyperelliptic (resp. nonhyperelliptic) curve. An irreducible curve $C$ on $S$ is vertical (with respect to $f$ ) if $f(C)$ is a point; otherwise, we say $C$ is horizonal.

(1.2) Let $f: S \rightarrow B$ be a relatively minimal fibration of genus $g \geq 2$, and $\sigma$ an involution of $S$ inducing the trivial action on $B$. Let $u: \tilde{S} \rightarrow S$ be the blowup of all isolated fixed points of $\sigma$, and $\tilde{\sigma}$ the induced involution on $\tilde{S}$. Let $P_{\sigma}=\tilde{S} / \tilde{\sigma}$. Then $f$ induces a fibration $h_{\sigma}: P_{\sigma} \rightarrow B$ of genus $g(F / \sigma)$ (not relatively minimal in general). We have a commutative diagram

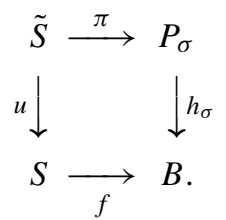

(1.2.1) If $\Gamma<f^{*} b(b \in B)$ is a $\sigma$-fixed curve, then from $(f \circ u)^{*} b=\pi^{*}\left(h_{\sigma}^{*} b\right)$, the coefficient of $\Gamma$ in $f^{*} b$ is divisible by 2 . In particular, if $f^{*} b$ is reduced, then $\sigma$ acts nontrivially on any irreducible component of $f^{*} b$.

Let $F^{\prime}$ be a semistable fiber of $f$ (i.e., $F^{\prime}$ is reduced with only nodes as singularities), and $p \in F^{\prime}$ a node. We say that $p$ is a separating point (resp. nonseparating point) of $F^{\prime}$, if $F^{\prime} \backslash\{p\}$ is disconnected (resp. connected) as a topological space.

(1.3) (cf. [Ca, Lemma 2.4]) Let $f: S \rightarrow B$ and $\sigma$ be as above, and $F^{\prime}$ a semistable singular fiber of $f$. If $p \in F^{\prime}$ is an isolated fixed point of $\sigma$, then $p$ is a node of $F^{\prime}$, and moreover if $\sigma$ is a hyperelliptic involution of $S$, then $p$ is a separating point of $F^{\prime}$.

(1.4) Notation as in (1.2). If $f$ is a relatively minimal hyperelliptic fibration, gluing the hyperelliptic involution of $F$ gives an everywhere defined involution $\sigma$ on $S$. Then $h_{\sigma}: P_{\sigma} \rightarrow$ $B$ is a ruled surface. Let $(\tilde{R}, \tilde{\delta})$ be the double cover data corresponding to $\pi: \tilde{S} \rightarrow P_{\sigma}$. One has a minimal ruled surface $P$, and a (possibly singular) double cover data $(R, \delta)$ on $P$, satisfying the following conditions:

(i) There is a birational morphism $\phi: P_{\sigma} \rightarrow P$ such that $\tilde{R}$ is the reduced inverse image of $R$; 
(ii) Let $R_{h}$ be the sum of the nonvertical irreducible components of $R$. Then the singularities of $R_{h}$ are at most of order $g+1$, and $R^{2}$ is the smallest among all such choices (cf. [X1, Lemma 6]). ( $P, R, \delta)$ is called the genus $g$ data corresponding to $f$.

(1.4.1) ([X1, Definition 5]) Let $f: S \rightarrow B$ be a hyperelliptic fibration corresponding to genus $g$ data $(P, R, \delta)$. For any fiber $F$ of $f$ and $i=3, \ldots, g+2$, we define the $i$-singularity $s_{i}(F)$ of $F$ as follows:

If $i$ is odd, $s_{i}(F)$ equals the number of singularities of type $i \rightarrow i$ (that is, infinitely near points of multiplicity $i$ ) of $R$ on the image of $F$.

If $i$ is even, $s_{i}(F)$ equals the number of singularities of order $i$ of $R$ on the image of $F$, not belonging to a singularity of type $i-1 \rightarrow i-1$ or $i+1 \rightarrow i+1$.

The singularities $s_{i}(F)$ do not depend on the choice of the contraction map $\phi: P_{\sigma} \rightarrow P$ (cf. [X1, Lemma 8]). Clearly there are only a finite number of fibers $F$ with $s_{i}(F) \neq 0$ for each $i$. A fiber $F$ is essential, if $s_{i}(F) \neq 0$ for some $i$.

(1.4.2) (Xiao [X1, Theorem 1]) Let $f: S \rightarrow B$ be the hyperelliptic fibration corresponding to genus $g$ data $(P, R, \delta)$. If $f$ has no essential fibers, then

$$
K_{S}^{2}=\frac{4 g-4}{g} \chi\left(\mathcal{O}_{S}\right)-\frac{4\left(g^{2}-1\right)(g(B)-1)}{g} .
$$

(1.5) (Reid [Re]) Let $f: S \rightarrow B$ be a nonhyperelliptic fibration of genus $g=3$. Then the natural morphism of sheaves

$$
r: S^{2}\left(f_{*} \omega_{S / B}\right) \rightarrow f_{*} \omega_{S / B}^{2}
$$

is generically surjective. Let $\mathcal{M}=$ Coker $r$. Then $\mathcal{M}=\bigoplus_{b \in B} \mathcal{M}_{b}$, where $\mathcal{M}_{b}$ is the stalk of $\mathcal{M}$ at $b \in B$, which is an $\mathcal{O}_{B, b}$-module of finite length. Let $H(S / B, b)=$ length $\mathcal{M}_{b}$. For any $b \in B$, if $f^{*} b$ is a smooth nonhyperelliptic curve or an irreducible nonhyperelliptic curve with one node whose normalization is a curve of genus 2 , then $H(S / B, b)=0$. Using the Riemann-Roch theorem on $S$ and the Leray spectral sequence, we have

$$
K_{S}^{2}=3 \chi\left(\mathcal{O}_{S}\right)+10(g(B)-1)+\sum_{b \in B} H(S / B, b) .
$$

For any normal surface $X$, we denote by $p_{g}(X)$ the geometric genus of a nonsingular model of $X$.

(1.6) (Beauville [Be]) Let $S$ be a projective minimal nonsingular surface of general type with $\chi\left(\mathcal{O}_{S}\right) \geq 21$, and $\phi_{S}: S--\rightarrow \boldsymbol{P}^{p_{g}(S)-1}$ the canonical map. There are two cases:

(1.6.1) $\phi_{S}$ is composed with a pencil. Then the moving part of $\left|K_{S}\right|$ is base point free. Let $f: S \rightarrow B$ be the fibration associated with $\phi_{S}$, and $g$ the genus of the general fiber of $f$. Then $2 \leq g \leq 5$ and $K_{S}^{2} \geq(2 g-2)\left(\chi\left(\mathcal{O}_{S}\right)-2\right)$.

(1.6.2) $\operatorname{dim} \operatorname{Im} \phi_{S}=2$. If $\chi\left(\mathcal{O}_{S}\right) \geq 31$, then either (i) $p_{g}\left(\operatorname{Im} \phi_{S}\right)=0$ and $\operatorname{deg} \phi_{S} \leq 9$ or (ii) $p_{g}\left(\operatorname{Im} \phi_{S}\right)=p_{g}(S)$ and $\operatorname{deg} \phi_{S} \leq 3$.

(1.7) (Xiao [X2]) Let $f: S \rightarrow B$ be as in (1.6.1). Then $g(B) \leq 1$. 
(1.8) (A special case of the logarithmic Miyaoka-Yau inequality. cf. [Sa]) Let $S$ be a projective nonsingular complex surface of general type and $C \subset S$ a nonsingular curve. Then $K_{S}^{2} \leq 9 \chi\left(\mathcal{O}_{S}\right)+(g(C)-1)-K_{S} C / 4$.

(1.9) (Accola [Ac]) Let $C$ be a curve of genus $g$, and $G \subset$ Aut $C$ a finite group. If $G$ admits a partition, i.e., $G=\bigcup_{i=1}^{s} G_{i}$, where $G_{i}$ are subgroups of $G$ satisfying $G_{i} \cap G_{j}=$ $\left\langle 1_{G}\right\rangle$ for all $i \neq j$, then

$$
(s-1) g+|G| g(C / G)=\sum_{i=1}^{s}\left|G_{i}\right| g\left(C / G_{i}\right) .
$$

For example, assume that $G=D_{2 n}$ is a dihedral group of order $2 n$. Let $\alpha \in G$ generate the cyclic subgroup of order $n$, and let $\beta \in G$ be an element of order 2 not in $\langle\alpha\rangle$. Then $\beta_{i}=\alpha^{i} \beta$ $(i=1,2, \ldots, n)$ are elements in $G$ not in $\langle\alpha\rangle$. So $G$ admits a partition and we have

$$
g+2 g(C / G)=g(C /\langle\alpha\rangle)+g\left(C /\left\langle\beta_{1}\right\rangle\right)+g\left(C /\left\langle\beta_{2}\right\rangle\right) .
$$

(1.10) Let $S$ be a smooth surface, $\sigma \in$ Aut $S$, and $p \in S$ a fixed point of $\sigma$. Then $\sigma$ induces a linear action on the tangent space $T_{p} S$ of $S$ at $p$. If this action is trivial, then $\sigma$ is trivial.

A curve $C \subset S$ is $\sigma$-invariant (resp. $\sigma$-fixed), if $\sigma(C)=C$ (resp. $\sigma(p)=p$ for any $p \in C)$.

(1.11) If a reduced $\sigma$-fixed curve $C$ is singular, then $\sigma$ is trivial. This follows from (1.10), since the induced action of $\sigma$ on the tangent space at the singular point of $C$ is trivial.

(1.12) Let $C$ be a curve of genus $g$, and $G \subset$ Aut $C$ a finite group. If $G$ has a fixed point, then $G$ is cyclic.

(1.13) Let $C$ be a curve of genus $g \geq 2$, and $G \subset$ Aut $C$ an abelian group. Assume that $g(C / G)=1$. Let $\pi: C \rightarrow C / G$ be the quotient map. Let $q_{i}(i=1, \ldots, k)$ be the points over which $\pi$ is ramified and $r_{i}$ the ramification number of $\pi$ over $q_{i}$. Then $k \geq 2$, and if $k=2$ then $r_{1}=r_{2}$. Indeed, $G$ is an abelian quotient of $\pi_{1}\left(C / G \backslash\left\{q_{1}, \ldots, q_{k}\right\}\right)$, which is generated by $\alpha, \beta, \gamma_{1}, \ldots, \gamma_{k}$ with one relation $\alpha \beta \alpha^{-1} \beta^{-1} \gamma_{1} \cdots \gamma_{k}=1$, where $\alpha$ and $\beta$ are generators of $\pi_{1}(C / G)$ and $\gamma_{i}$ is a small loop around $q_{i}$. Let $\bar{\gamma}_{i}$ be the image of $\gamma_{i}$ in $G$. Then $\bar{\gamma}_{i}$ is of order $r_{i}$ and $\bar{\gamma}_{1} \cdots \bar{\gamma}_{k}=1$.

(1.14) Let $S$ be a smooth projective surface, and $G \subset$ Aut $S$ a finite subgroup such that $G$ acts trivially on $H^{2}(S, Q)$. By the argument of [Pe, Lemma 2], we have that, if $p \in S$ a $\sigma$-fixed point for some id $\neq \sigma \in G$, then either $p \in \mathrm{Bs}\left|K_{S}\right|$ (the base locus of $\left|K_{S}\right|$ ) or $p$ is an isolated $\sigma$-fixed point. This implies:

(1.14.1) If $C \subset S$ is a $\sigma$-fixed curve for some id $\neq \sigma \in G$, then $C \subset \mathrm{Bs}\left|K_{S}\right|$.

(1.14.2) If $C \subset S$ is a $G$-invariant curve, and $C \not \subset \mathrm{Bs}\left|K_{S}\right|$, then $G$ acts faithfully on $C$, i.e., $G \hookrightarrow$ Aut $C$.

(1.15) Let $S$ and $G$ be as in (1.14). Assume that $S$ has a fibration $f: S \rightarrow B$ and $G$ induces the trivial action on $B$. If $p_{g}(S)>0$ then $g(F / G)>0$, where $F$ is a general fiber of $f$. Indeed, We have $p_{g}(S / G)=\operatorname{dim} H^{0}\left(S, \omega_{S}\right)^{G}$ (cf. [Fr, p. 99]). By Hodge theory, 
$H^{0}\left(S, \omega_{S}\right)^{G}=H^{0}\left(S, \omega_{S}\right)$. So $p_{g}(S / G)=p_{g}(S)$ and thus the general fiber of $S / G \rightarrow B$ is not rational if $p_{g}(S)>0$.

2. First reductions. To prove Theorem A, let me start by fixing notation.

(2.1) Let $S$ be a complex minimal nonsingular projective surface of general type with $\chi\left(\mathcal{O}_{S}\right) \geq 21$. Assume that the canonical map $\phi_{S}$ of $S$ is composed with a pencil.

Let $G \subset$ Aut $S$ be a subgroup of automorphisms of $S$, inducing trivial actions on $H^{2}(S, Q)$.

Let $M$ and $Z$ be the moving part and the fixed part of $\left|K_{S}\right|$, respectively. By (1.6.1), $|M|$ has no base points. Let

$$
\phi_{S}=\varphi \circ f: S \rightarrow B \rightarrow \operatorname{Im} \phi_{S} \subset \boldsymbol{P}^{p_{g}(S)-1}
$$

be the Stein factorization of $\phi_{S}$. We call $f: S \rightarrow B$ the canonical fibration associated with $\phi_{S}$. Let $F$ be a general fiber of $f$, and $g$ the genus of $F$.

Let $d$ and $L$ be the degree and the hyperplane section of $\operatorname{Im} \phi_{S}$ in $\boldsymbol{P}^{p_{g}(S)-1}$ respectively. We have $\mathcal{O}_{S}(M)=f^{*} \varphi^{*} L$ and $M \sim_{\text {num }} \operatorname{deg} \varphi d F$. Note that $h^{1}\left(B, \varphi^{*} L\right)=0$, since $g(B) \leq$ 1 by (1.7), and $d \geq \operatorname{codim} \operatorname{Im} \phi_{S}+1$ (cf. [Mu]). From

$$
p_{g}(S)=h^{0}\left(S, \varphi^{*} L\right)=\operatorname{deg}\left(\varphi^{*} L\right)+1-g(B)+h^{1}\left(B, \varphi^{*} L\right)=\operatorname{deg} \varphi d+1-g(B),
$$

we get

$$
\begin{aligned}
& \operatorname{deg} \varphi=1 \text { and } \\
& d=\left\{\begin{array}{l}
\chi\left(\mathcal{O}_{S}\right) \quad \text { if } g(B)=1, \\
\chi\left(\mathcal{O}_{S}\right)-2+q(S) \quad \text { if } g(B)=0 .
\end{array}\right.
\end{aligned}
$$

(2.2) Since $H^{0}\left(S, \omega_{S}\right)$ is a direct factor of $H^{2}(S, C)$ by Hodge theory, $G$ acts trivially on $H^{0}\left(S, \omega_{S}\right)$. This implies that $G$ acts trivially on $\operatorname{Im} \phi_{S}$ and there is a homomorphism $h$ of $G$ into Aut $B$. Since $\operatorname{deg} \varphi=1$ (2.1.1), we have that $\operatorname{Ker} h=G$, i.e., $G$ induces the trivial action on $B$, and $G \hookrightarrow$ Aut $F$ for a general fiber $F$ of $f$.

Notation 2.3. Let $f: S \rightarrow B$ and $G$ be as above.

(i) We write $Z=H+V$ and $H=n_{1} \Gamma_{1}+n_{2} \Gamma_{2}+\cdots$ with $n_{1} \geq n_{2} \geq \cdots$, where $H$ (resp. $V$ ) is the horizontal part (resp. the vertical part) of $Z$, and $\Gamma_{i}(i=1,2, \ldots$ ) are the irreducible components of $H$, with $n_{i}$ the multiplicity of $\Gamma_{i}$ in $H$.

(ii) For a general fiber $F$ of $f$, let $R_{F}$ be the set of ramified points of the quotient map $F \rightarrow F / G$. For any two curves $C$ and $D$ on $S$, we denote by $C \cap D$ the set-theoretic intersection supp $C \cap \operatorname{supp} D$.

LEMMA 2.4. Let $f: S \rightarrow B, H, \Gamma_{i}$ and $G$ be as in (2.1). Let $F$ be a general fiber of f. Then

(2.4.1) $\quad R_{F} \subset H \cap F$.

(2.4.2) If $R_{F}=H \cap F$, then $\Gamma_{i}$ is smooth for every $i$. 
Proof. (i) Suppose that there is a point $p \in F$ such that $p \in R_{F}$ and $p \notin H \cap F$. Then there exists an element id $\neq \sigma \in G$ such that $p$ is $\sigma$-fixed. Since $F$ is a general fiber, $p$ is not an isolated fixed point of $\sigma$. So there exists a $\sigma$-fixed curve $C$ passing through $p$. By (1.14.1), $C \subset \mathrm{Bs}\left|K_{S}\right| . C$ is not vertical since $F$ is a general fiber. So $C<H$, which contradicts the assumption that $p \notin H \cap F$.

(ii) For a general point $p \in \Gamma_{i}, p \in H \cap f^{*}(f(p))=R_{f^{*}(f(p))}$. This implies there exists id $\neq \sigma_{p} \in G$ such that $p$ is $\sigma_{p}$-fixed. Since $G$ is finite, there is a id $\neq \sigma \in G$ such that $\Gamma_{i}$ is $\sigma$-fixed. So $\Gamma_{i}$ is smooth by (1.11).

LEMMA 2.5. Let $f: S \rightarrow B, H, g$ and $G$ be as in (2.1). Let $F$ be a general fiber of $f$. If $2 \leq g \leq 4$, then either $|G| \leq 2 g-2$, or $G$ is nonabelian, $G$ acts transitively on $H \cap F^{\prime}$ for any fiber $F^{\prime}$ of $f$, and the only possibilities for the triple $(g,|G|, \#(H \cap F))$ are as follows:

$$
(3,8,4), \quad(3,6,2), \quad(4,12,6), \quad(4,8,2) \text {. }
$$

Moreover, if $(g,|G|, \#(H \cap F))=(3,8,4)$ or $(4,12,6)$, then $H$ is reduced and each irreducible component of $H$ is smooth.

Proof. For any point $p \in S$, let $\operatorname{stab}(p)=\{\tau \in G \mid \tau(p)=p\}$. If $r_{p}:=|\operatorname{stab}(p)|=1$ for some $p \in H \cap F$, then $|G| \leq \#(H \cap F) \leq 2 g-2$. So we can assume that $|\operatorname{stab}(p)| \geq 2$ for each $p \in H \cap F$. Let $m$ be the number of orbits of $H \cap F$ under the action of $G$. Then by (2.4.1), the quotient map $\pi: F \rightarrow F / G$ has exactly $m$ branch points. Using the Hurwitz formula for $\pi$, we get $|G| \leq 2 g-2$ if either $g(F / G) \geq 2$, or $g(F / G)=1$ and either $G$ is abelian or $m \geq 2$. Hence we can assume that $g(F / G)=1(g(F / G) \neq 0$ by (1.15)), $G$ is nonabelian and $m=1$. Then $G$ acts transitively on $H \cap F$ and hence on $H \cap F^{\prime}$ for any fiber $F^{\prime}$ of $f$. In this case, for any point $p \in H \cap F$, we have $|G| / r_{p}=\#(H \cap F)$ and $\#(H \cap F) \mid 2 g-2$. Using the Hurwitz formula for $\pi$ again, we have $|G|=\#(H \cap F)+2 g-2$. Note that $G$ is nonabelian in this case, and we get that $(g,|G|, \#(H \cap F))$ equals one of the triples listed in the lemma. The last statement follows by (2.4.2).

REMARK 2.6. If $(g,|G|, \#(H \cap F))=(3,6,2)$, then either $H=2 \Gamma_{1}+2 \Gamma_{2}$ or $H=2 \Gamma$, where $\Gamma_{i}$ are sections of $f$ and $\Gamma$ is an irreducible smooth curve with $\Gamma F=2$.

PROPOSITION 2.7. Let $S$ be a complex minimal nonsingular projective surface of general type with $\chi\left(\mathcal{O}_{S}\right) \geq 21$, and let $G \subset$ Aut $S$ be a subgroup of automorphisms of $S$ inducing trivial actions on $H^{2}(S, Q)$. Assume that the canonical map $\phi_{S}$ is composed with a pencil. Let $f: S \rightarrow B$ be the canonical fibration associated with $\phi_{S}$, and $g$ the genus of a general fiber of $f$. Furthermore, assume $\chi\left(\mathcal{O}_{S}\right)>188$ if $g=4$, and $\chi\left(\mathcal{O}_{S}\right)>60$ if $g=5$. Then $|G| \leq 4$.

Proof. By (1.6.1), $2 \leq g \leq 5$. If $g=2$, we have $|G| \leq 2$ by Lemma 2.5. The proof of the case $3 \leq g \leq 5$ is longer and is postponed till the next two sections.

Proof of Theorem A. By Proposition 2.7, we can assume that $\phi_{S}$ is generically finite. Since $H^{0}\left(S, \omega_{S}\right)$ is a direct factor of $H^{2}(S, \boldsymbol{Q})$ by Hodge theory, $G$ acts trivially on $H^{0}\left(S, \omega_{S}\right)$. This implies that $G$ induces trivial actions on $\operatorname{Im} \phi_{S}$. So $\phi_{S}$ factors through the 
quotient map

$$
\phi_{S}=\alpha \circ q: S \stackrel{q}{\longrightarrow} S / G \stackrel{\alpha}{\longrightarrow} \operatorname{Im} \phi_{S} .
$$

Thus $|G|=\operatorname{deg} \phi_{S} / \operatorname{deg} \alpha$. Now if $S$ is as in case (ii) of (1.6.2), then $|G| \leq 3$. If $S$ is as in case (i) of (1.6.2), then $\operatorname{deg} \alpha \geq 2$, since $p_{g}(S / G)=p_{g}(S) \neq 0=p_{g}\left(\operatorname{Im} \phi_{S}\right)$. So $|G| \leq \operatorname{deg} \phi_{S} / 2 \leq 9 / 2$.

\section{Proof of Proposition 2.7, the case $g=3$.}

LEMMA 3.1. Let $S$ be a complex nonsingular projective surface, and $G \subset$ Aut $S$ a subgroup of automorphisms of $S$ inducing trivial actions on $H^{2}(S, Q)$. Let $C \subset S$ be an irreducible curve. If $C^{2}<0$, then $C$ is $G$-invariant.

Proof. Indeed, if $C$ is not $\sigma$-invariant for some id $\neq \sigma \in G$, then $\left(\sigma^{*} C\right) C \geq 0$. On the other hand, $\sigma^{*} C$ is numerically equivalent to $C$, since $G$ acts trivially on $\operatorname{NS}(S) \otimes Q \hookrightarrow$ $H^{2}(S, Q)$. So $\left(\sigma^{*} C\right) C=C^{2}<0$, a contradiction.

Lemma 3.2. Let $f: S \rightarrow B, H, g$ and $G$ be as in (2.1). Assume that $g=3$ and $G$ is a nonabelian group of order 8.

(i) Let $\sigma$ be the generator of the center of $G$, which is clearly a cyclic subgroup of order 2. Then $H$ is $\sigma$-fixed (and hence smooth), and $G /\langle\sigma\rangle \hookrightarrow$ Aut $H$.

(ii) Let $F^{\prime}$ be a singular fiber of $f$ and $C<F^{\prime}$ an irreducible component with $C H \neq 0$. Then $G \hookrightarrow$ Aut $C$.

Proof. (i) Let $F$ be a general fiber of $f$. Let $\bar{F}=F /\langle\sigma\rangle$ and $\bar{G}=G /\langle\sigma\rangle$. Since $g(F / G)=1$ and $|\bar{G}|=4$, using the Hurwitz formula for $\bar{F} \rightarrow \bar{F} / \bar{G} \simeq F / G$, we get $g(\bar{F})=1$. So $\sigma$ has four fixed points on $F$. Since $\#(H \cap F)=4$ in Lemma 2.5, by (2.4.1), $H$ is $\sigma$-fixed and hence smooth by (1.11). Since $G$ acts transitively on $H \cap F$ and $\#(H \cap F)=4$, we have $G /\langle\sigma\rangle \hookrightarrow$ Aut $H$.

(ii) If $F^{\prime}$ is reducible, $C$ is $G$-invariant by (3.1); if the reduced scheme $F_{\text {red }}^{\prime}$ of $F^{\prime}$ is irreducible, then $C=F_{\text {red }}^{\prime}$ is clearly $G$-invariant. So there is a homomorphism $h: G \rightarrow$ Aut $C$.

Let $\sigma$ be as in (i). If $\sigma \in \operatorname{Ker} h$, then $C+H$ is $\sigma$-fixed. So $\sigma$ is trivial by (1.11). This is impossible. Hence the lemma follows by showing that $\sigma \in \operatorname{Ker} h$ if $\operatorname{Ker} h$ is not trivial.

Suppose that Ker $h$ is not trivial. If $G \simeq Q_{8}$, we have that $\sigma \in \operatorname{Ker} h$ since there is only one element of order 2 in $Q_{8}$. Now assume that $G \simeq D_{8}$. If $|\operatorname{Ker} h|=2$, we get $\operatorname{Ker} h=\langle\sigma\rangle$ since a normal subgroup of order 2 must be contained in the center of $G$; If $|\operatorname{Ker} h|=4$, let $\alpha \in G$ be an element of order 4. Then $\sigma=\alpha^{2}$ and $h\left(\alpha^{2}\right)=h(\alpha)^{2}=$ id. So $\sigma \in \operatorname{Ker} h$.

LEMMA 3.3. (i) Let $G$ be a nonabelian group of order 8. Assume that $G \hookrightarrow$ Aut $C$ for some smooth curve $C$ of genus $\leq 1$. Then $G \simeq D_{8}$. Moreover, if $g(C)=1$, the elements of order 4 of $G$ act freely on $C$.

(ii) If $G \simeq D_{6} \hookrightarrow$ Aut $C$ for some smooth elliptic curve $C$, then the elements of order 3 of $G$ act freely on $C$. 
Proof. (i) If $C \simeq \boldsymbol{P}^{1}$, the lemma follows by the well known fact that a finite subgroup of Aut $\boldsymbol{P}^{1}$ is isomorphic to one of the following groups: $C_{n}, D_{2 n}, T_{12}, O_{24}$ and $I_{60}$, where $T_{12}, O_{24}$ and $I_{60}$ are the polyhedral groups of indicated orders.

If $C$ is an elliptic curve, then $G=T \rtimes A$ (a semi-direct product), where $T$ is a group of translations and $A \subset$ Aut $C$ is a subgroup preserving the group structure. If $T \simeq C_{2}$, then $G$ must be abelian, which contradicts the assumption. Now assume that $|T|=4$. Let $\alpha \in G$ be an element of order 4. Then it is easy to see that $\alpha^{2} \in T$ since $|A|=2$ in this case. So $\alpha^{2}$ and hence $\alpha$ has no fixed points. This implies $\alpha \in T$. Hence $T \simeq C_{4}$, and the result follows.

(ii) follows by an argument similar to that in (i).

LEMMA 3.4. Let $f: S \rightarrow B, g$ and $G$ be as in (2.1). Assume that $g=3$.

(i) If $G \simeq Q_{8}, f$ is nonhyperelliptic.

(ii) If $G \simeq D_{8}$ or $D_{6}, f$ is hyperelliptic.

Proof. (i) Otherwise, let $\tau$ be the hyperelliptic involution of a general fiber $F$ of $f$. Since $g(F / G)=1$ by (1.15), we get $\tau \notin G$. This implies $G \hookrightarrow$ Aut $\boldsymbol{P}^{1}$, since Aut $F$ is a $\langle\tau\rangle$-extension of a subgroup of Aut $\boldsymbol{P}^{1}$. This is impossible by Lemma 3.3.

(ii) Let $F$ be a general fiber of $f$. If $G \simeq D_{2 n}$ for $n=3$ or 4 , then by (1.9) we have $g(F)+2 g\left(F / D_{2 n}\right)=g(F /\langle\alpha\rangle)+g\left(F /\left\langle\beta_{1}\right\rangle\right)+g\left(F /\left\langle\beta_{2}\right\rangle\right)$, where $\alpha$ and $\beta_{i}$ are as in (1.9). Since $g\left(F / D_{2 n}\right)=1$ and $g(F /\langle\alpha\rangle)=1$, we get $g\left(F /\left\langle\beta_{i}\right\rangle\right)=2$. So $F$ is étale over a curve of genus 2 . This implies $F$ is hyperelliptic by [Ac].

LEMMA 3.5. Let $f: S \rightarrow B$ be a nonhyperelliptic fibration of genus 3 , and $G \subset$ Aut $S$ a subgroup inducing the trivial action on $B$. Let $F^{\prime}$ be a fiber of $f$. Assume that $G \simeq Q_{8}$, and that $F^{\prime}$ is either a smooth hyperelliptic curve or a multiple fiber $2 C$ with $C$ smooth of genus 2. Then the kernel of the homomorphism $h: G \rightarrow$ Aut $F_{\text {red }}^{\prime}$ is not trivial.

Proof. Suppose that ker $h$ is trivial. Denote by $\sigma$ the unique element of order 2 in $G$.

First we assume that $F^{\prime}=2 C$, where $C$ is a smooth curve of genus 2 . Let $p^{\prime}=f\left(F^{\prime}\right)$ and fix a point $p \in B$ such that $f^{*} p$ is smooth. Let $\tilde{B} \rightarrow B$ be a double cover ramified exactly at $p$ and $p^{\prime}$, and let $\pi^{\prime}: \tilde{S} \rightarrow \tilde{B} \times{ }_{B} S$ be the normalization. Then $\pi:=p_{2} \circ \pi^{\prime}: \tilde{S} \rightarrow S$ is ramified along $f^{*} p$, and $\tilde{f}:=p_{1} \circ \pi^{\prime}: \tilde{S} \rightarrow \tilde{B}$ is a fibration of genus 3 , where $p_{1}$ and $p_{2}$ are the projections of $\tilde{B} \times{ }_{B} S$ onto its factors. Let $\tilde{p}^{\prime}$ be the inverse image of $p^{\prime}$. Then $\tilde{F}^{\prime}:=\tilde{f}^{*} \tilde{p}^{\prime}$ is a smooth hyperelliptic curve. Since $G$ induces the trivial action on $B, \tilde{B} \times{ }_{B} S \subset \tilde{B} \times S$ is $G$-invariant. So $G$ acts on $\tilde{S}$, inducing the trivial action on $\tilde{B}$. We have $G \hookrightarrow$ Aut $\tilde{F}^{\prime}$ if $\operatorname{Ker} h$ is trivial. Hence the lemma is reduced to the case when $F^{\prime}$ is a smooth hyperelliptic curve.

Now assume that $F^{\prime}$ is a smooth hyperelliptic curve. Let $\tau$ be the hyperelliptic involution of $F^{\prime}$. If $\tau \notin G$, then $G \hookrightarrow$ Aut $\boldsymbol{P}^{1}$ since Aut $F^{\prime}$ is a $\langle\tau\rangle$-extension of a subgroup of Aut $\boldsymbol{P}^{1}$. This is impossible by Lemma 3.3. So we can assume that $\sigma$ is the hyperelliptic involution of $F^{\prime}$. Then there are eight $\sigma$-fixed points on $F^{\prime}$. By (1.3), there exists a $\sigma$-fixed curve $D$ passing through these points. Since $G \simeq Q_{8} \hookrightarrow$ Aut $F^{\prime}$ by assumption, we get $F^{\prime} \neq D$. Now for a general fiber $F$, there are at least $\#(D \cap F)=D F=D F^{\prime} \geq 8 \sigma$-fixed points. 
This implies that $\sigma$ is the hyperelliptic involution of $F$, contradicting the assumption that $f$ is nonhyperelliptic.

Lemma 3.6. Let $f: S \rightarrow B, H, g$ and $G$ be as in (2.1). Assume that $g=3$ and $G$ is a nonabelian group of order 8 . Let $F^{\prime}$ be a singular fiber of $f$ and $C<F^{\prime}$ an irreducible component. Denote by $\tilde{C}$ the normalization of $C$. If $g(\tilde{C}) \geq 2$, then $F^{\prime}$ belongs to one of the following possible types.

(i) $F^{\prime}=2 C$, and $C$ is smooth;

(ii) $F^{\prime}=C$ is an irreducible curve with one node, and the normalization of $F^{\prime}$ is a curve of genus 2 ;

(iii) $F^{\prime}=C+D$, where $C$ and $D$ are irreducible smooth curves meeting transversally at two points, and $g(C)=2$ and $g(D)=0$.

Proof. We have either $p_{a}(C)=3$ or $C=\tilde{C}$. In the former case, $F^{\prime}=C$ is an irreducible curve with one singularity, say $q$, and its normalization is a curve of genus 2 . If $q \in F^{\prime}$ is a cusp, the inverse image $\tilde{q}$ of the cusp $q \in F^{\prime}$ under the normalization map is $G$-fixed. This implies $G$ is cyclic by (1.12), a contradiction. So $F^{\prime}$ is of type (ii). In the latter case, since $K_{S} C=2-C^{2} \geq 2$ and $K_{S} F^{\prime}=4$, we get either $C^{2}=0\left(F^{\prime}\right.$ is of type (i)) or mult ${ }_{C} F^{\prime}=1$. Now assume that mult $_{C} F^{\prime}=1$. Then $F^{\prime}$ is 1 -connected. Let $D<F^{\prime}$ be an irreducible curve such that $D C>0$. If $\#(D \cap C)=1, G$ is cyclic, a contradiction. So $\#(D \cap C) \geq 2$ and hence $D C \geq 2$. Note that $p_{a}(D+C) \leq 3$, hence we have $D C=2$ and $F^{\prime}$ is of type (iii).

Proof of Proposition 2.7, The CASE $g=3$. Let $f: S \rightarrow B$ be the canonical fibration associated with $\phi_{S}$. By (2.2), $G$ induces the trivial action on $B$, and $G \hookrightarrow$ Aut $F$, where $F$ is a general fiber of $f$. Assume $g=3$. By Lemma 2.5, if $|G|>4$, then $G$ is isomorphic to $Q_{8}, D_{8}$ or $D_{6}$. Now the result follows by the next claims.

CLAIM 3.7. $G \simeq Q_{8}$ does not occur.

Proof. Suppose $G \simeq Q_{8}$. Then by Lemma 3.4, $f$ is nonhyperelliptic. Since $g(B) \leq 1$ by (1.7), $f$ has singular fibers.

Let $F^{\prime}$ be a singular fiber of $f$, and let $C<F^{\prime}$ be an irreducible component such that $C H \neq 0$. By Lemma 3.2 (ii), we have $G \hookrightarrow$ Aut $C$. By Lemmas 3.3 (i), 3.5 and 3.6, we have that $F^{\prime}$ is of type (ii) or (iii) of (3.6).

If $F^{\prime}$ is of type (iii) of (3.6), we have that either $D \nless V$ or $H D>0$, where $V$ is as in (2.1). Indeed, if both $D<V$ and $H D=0$ hold, then $C \nless V$ and thus $V D<0$. But from $0=K_{S} D=(M+H+V) D$, we get $V D=0$, a contradiction. Now by Lemma 3.2 (ii) and (1.14.2), $Q_{8} \hookrightarrow$ Aut $D$. This is impossible by Lemma 3.3 (i).

Now if $F^{\prime}$ is of type (ii) of Lemma 3.6, we show that $F^{\prime}$ is nonhyperelliptic.

Let $\sigma$ be the generator of the center of $G$. We have $G /\langle\sigma\rangle \simeq C_{2} \times C_{2}$. First we claim that the node $q \in F^{\prime}$ is an isolated $\sigma$-fixed point. Otherwise, there is a $\sigma$-fixed curve $D$ passing through $q$. By (1.14.1), $D<H$. Since $q \in F^{\prime}$ is $G$-fixed, $q \in H$ is $G /\langle\sigma\rangle$-fixed. By Lemma 3.2 (i) and (1.12), $G /\langle\sigma\rangle$ is cyclic, a contradiction. 
Second, we claim that $\sigma$ preserves the local two branches at $q$. Indeed, let $G^{\prime} \subset G$ be the subgroup preserving the local two branches at $q$. Clearly $G^{\prime}$ is cyclic of order 4 . Let $\alpha$ be a generator of $G^{\prime}$. If $\sigma \notin G^{\prime}$, then $\sigma$ and $\alpha$ generate $G$, and it is easy to see that $\sigma \alpha \sigma=\alpha^{-1}$. This implies that $G \simeq D_{8}$, a contradiction.

Now we have that $q$ is an isolated $\sigma$-fixed point and that $\sigma$ preserves the local two branches at $q$. So $h_{\sigma}^{*}(f(q))$ consists of two irreducible smooth curves meeting transversally at two points, where $h_{\sigma}: P_{\sigma} \rightarrow B$ is as in (1.2). Since $h_{\sigma}$ is of genus $1, \sigma$ is a hyperelliptic involution of the normalization $\tilde{F}^{\prime}$ of $F^{\prime}$. This implies that $F^{\prime}$ is a nonhyperelliptic fiber. Indeed, if there exists an involution $\tau$ on $F^{\prime}$ such that $F^{\prime} /\langle\tau\rangle \simeq \boldsymbol{P}^{1}$, then $\tau$ exchanges the local two branches at $q$, and $\tau$ is a hyperelliptic involution of $\tilde{F}^{\prime}$. This implies that $\sigma=\tau$ on $F^{\prime}$, which is absurd since one preserves the local two branches at $q$ while the other not.

By the above argument, we have that any singular fiber $F^{\prime}$ of $f$ is a nonhyperelliptic irreducible curve with one node. By Lemma 3.5 and (1.14.2), $f$ has no smooth hyperelliptic fibers. Thus $f$ has no fibers $F^{\prime}$ with non-vanishing $H\left(S / B, f\left(F^{\prime}\right)\right)$ (see (1.5) for the notation). By (1.5), we have that

$$
K_{S}^{2}=3 \chi\left(\mathcal{O}_{S}\right)+10(g(B)-1) .
$$

We get a contradiction by (1.6.1).

Claim 3.8. $G \simeq D_{8}$ or $D_{6}$ does not occur.

Proof. Suppose $G \simeq D_{6}$. The proof of the case $G \simeq D_{8}$ is similar and is left to the reader. By Lemma 3.4, $f$ is hyperelliptic. We will show that

(3.8.1) any singular fiber of $f$ belongs to one of the following types:

(i) $F^{\prime}=C$ is an irreducible curve with one node, and the normalization of $F^{\prime}$ is a curve of genus 2;

(ii) $F^{\prime}=C$ is an irreducible curve with three nodes, and the normalization of $F^{\prime}$ is isomorphic to $\boldsymbol{P}^{1}$;

(iii) $\quad F^{\prime}=C+D$, where $C$ and $D$ are irreducible smooth curves meeting transversally at two points, and $g(C)=2$ and $g(D)=0$.

We note that, if $F^{\prime}$ belongs to one of the types (i)-(iii), the singularities $s_{i}\left(F^{\prime}\right)=0$ for $i \geq 3$ (see (1.4.1) for the definition). (We check it when $F^{\prime}$ is of the type (iii); the other cases are similar. Let $q_{1}$ and $q_{2}$ be nodes of $F^{\prime}=C+D$. Let $\tau$ be the hyperelliptic involution of $f$. Let the notation be as in (1.2) and (1.4). Since the dual graph of $h_{\tau}\left(f\left(F^{\prime}\right)\right)$ is a tree, we have $\tau q_{1}=q_{2}$. Let $\bar{C}$ and $\bar{D}$ be the images of $C$ and $D$ under $\pi$, respectively. Then $h_{\tau}\left(f\left(F^{\prime}\right)\right)=\bar{C}+\bar{D}$ consists of two smooth rational curves meeting transversally at one point, and $\tilde{R}$ meets $\bar{C}$ (resp. $\bar{D}$ ) transversally at six (resp. two) points. By the choice of $\phi: P_{\tau} \rightarrow P$, $\phi$ contracts $\bar{D}$. Thus there is only one singular point of order 2 of $R$ on the image of $F^{\prime}$ and hence by (1.4.1) $s_{i}\left(F^{\prime}\right)=0$ for $i \geq 3$.)

Admitting (3.8.1) for the moment, we have that $f$ has no essential fibers, and hence by (1.4)

$$
K_{S}^{2}=\frac{8}{3} \chi\left(\mathcal{O}_{S}\right)-\frac{32(g(B)-1)}{3} .
$$


On the other hand, by (1.6.1), $K_{S}^{2} \geq 4\left(\chi\left(\mathcal{O}_{S}\right)-2\right)$, a contradiction.

It remains to prove (3.8.1). Let $\alpha \in D_{6}$ (resp. $\sigma \in D_{6}$ ) be an element of order 3 (resp. 2). By the proof of Lemma 2.5, $H$ is $\alpha$-fixed. Let $F^{\prime}$ be a singular fiber of $f$. Let $C<F^{\prime}$ be an irreducible component such that $C H \neq 0$, and $\tilde{C}$ the normalization of $C$. Then $C$ is $\alpha$-invariant by Lemma 3.1, and the homomorphism $h$ of $G$ into Aut $C$ is injective. (Otherwise, $\operatorname{Ker} h=\langle\alpha\rangle$ or $G$ since the nontrivial normal subgroup of $G$ is $\langle\alpha\rangle$. Hence $\alpha$ is trivial on $C+H$, which is impossible by (1.11).) We distinguish two cases according to whether $f_{H}: H_{\text {red }} \rightarrow B$ is étale at $H \cap F^{\prime}$ or not.

Case 1. $f_{H}$ is étale at $H \cap F^{\prime}$. In this case $H \cap F^{\prime}$ consists of two points, say $p_{1}$ and $p_{2}$. Since $H F^{\prime}=4$, by Remark $2.6, F^{\prime}$ is smooth at these points. Since $H$ is $\alpha$-fixed, $p_{1}$ and $p_{2}$ are $\alpha$-fixed. By the choice of $C$, there are at least two $\alpha$-fixed points $\left(p_{1}\right.$ and $\left.p_{2}\right)$ on it, and $C$ is smooth at $p_{i}$ for $i=1$ and 2 .

If $g(\tilde{C})=2$, then by the proof of Lemma 3.6, we have that $F^{\prime}$ is (i) or (iii).

If $g(\tilde{C})=1$, then by Lemma 3.3 (ii), we get a contradiction.

Now we assume $g(\tilde{C})=0$. We show that in this case either $F^{\prime}$ is of type (ii) or there exists a $\sigma$-fixed point $p \in C$ with $2 \nmid$ mult $_{p} F^{\prime}$. We consider three cases according to the singularities of $C$.

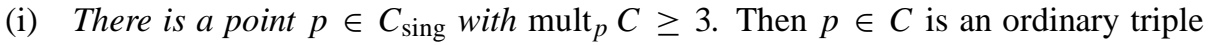
point and $C \backslash\{p\}$ is smooth and $F^{\prime}=C$. So $p$ is $\sigma$-fixed and mult $F_{p}^{\prime}=3$.

(ii) $C_{\text {sing }} \neq \emptyset$ and for any point $p \in C_{\text {sing, with mult }} C=2$. Since $p_{1}$ and $p_{2}$ are $\alpha$-fixed and $\alpha$ has exactly two fixed points on $\tilde{C} \simeq \boldsymbol{P}^{1}$, we have $\alpha(p) \neq p$ if $p \in C_{\text {sing. Hence }}$ either $F^{\prime}$ is of type (ii), or $F^{\prime}=C$ is an irreducible curve with three cusps (say $q_{1}, q_{2}$ and $q_{3}$ ) and the normalization of $F^{\prime}$ is isomorphic to $\boldsymbol{P}^{1}$. In the latter case, let $\tilde{q}_{i}(i=1,2,3)$ be the inverse image of $q_{i}$ under the normalization map $\tilde{C} \rightarrow C$. Since $\left\{q_{1}, q_{2}, q_{3}\right\}$ is $\sigma$-invariant and there are exactly two $\sigma$-fixed points on $\tilde{C} \simeq \boldsymbol{P}^{1}$, there must be a point $\tilde{p} \in \tilde{C} \backslash\left\{q_{1}, q_{2}, q_{3}\right\}$ which is $\sigma$-fixed. Let $p$ be the image of $\tilde{p}$ under the normalization map. Then $p$ is $\sigma$-fixed and mult $_{p} F^{\prime}=1$.

(iii) $C$ is a smooth rational curve. Since $F^{\prime}$ is 1-connected, there is an irreducible curve $D<F^{\prime}$ such that $D C>0$. Since $D \cap C$ is $\alpha$-invariant by Lemma 3.1, if \# $(D \cap C) \not \equiv$ $0(\bmod 3), \alpha$ has at least there fixed points $\left(p_{1}, p_{2}\right.$ and a point in $\left.D \cap C\right)$ on $C$. This implies $\alpha$ is trivial on $C$ and hence on $C+H$, which is impossible by (1.11). So we can assume $\#(D \cap C) \equiv 0(\bmod 3)$. Since $p_{a}(C+D) \leq 3$, we have $D C \leq 4$. So $\#(D \cap C)=3$. Since $D \cap C$ is $\sigma$-invariant and there are exactly two $\sigma$-fixed points on $\tilde{C} \simeq \boldsymbol{P}^{1}$, there is a point $p \in C \backslash D \cap C$ which is $\sigma$-fixed. We claim that mult $F_{p} F^{\prime}=1$. Otherwise, there is an irreducible curve $D^{\prime}<F^{\prime}$ passing through $p$. By the above argument, we can assume that $\#\left(D^{\prime} \cap C\right) \equiv 0(\bmod 3)$. This implies $p_{a}\left(C+D+D^{\prime}\right)>3$, a contradiction.

Now by the above argument, we have that either $F^{\prime}$ is of type (ii) or there is a $\sigma$-fixed point $p \in C$ with $2 \nmid$ mult $_{p} F^{\prime}$. In the latter case, let $u: \tilde{S} \rightarrow S$ be as in (1.2). If $p$ is an isolated $\sigma$-fixed point, then the inverse image $E=u^{-1}(p)$ of $p$ is a $\sigma$-fixed $(-1)$-curve, and the coefficient of $E$ in $(f \circ u)^{*}\left(f\left(F^{\prime}\right)\right)$ is not divisible by 2. This is impossible by (1.2.1). So there is a $\sigma$-fixed curve $D$ passing through $p$. Clearly $D \neq C$ by (1.11). By (1.14.1), 
$D<\mathrm{Bs}\left|K_{S}\right|$, and hence $D<H$. Since $\alpha$ and $\sigma$ generate $G$, this implies there is a $G$-fixed point $p^{\prime} \in H \cap F$, and thus $G$ is cyclic by (1.12), a contradiction.

Case 2. $f_{H}$ is not étale at $H \cap F^{\prime}$. Then $H \cap F^{\prime}$ consists of one point, say $p$. By the choice of $C, C$ passes through $p$. Since $G \hookrightarrow$ Aut $C$, by (1.12), $p \in C$ is a singular point.

Since $H F^{\prime}=4$, we have mult ${ }_{C} F^{\prime}=1$ and mult $_{p} C=2$. If $p \in C$ is a cusp, it is easy to see $G$ is cyclic by (1.12). So we can assume that $p \in C$ is a node. Blowup $S$ at $p$, and let $E$ be the exceptional curve and $\tilde{H}$ the strict transform of $H$. If $p$ is an ordinary node of $C$, then $\alpha$ preserves the local branches of $C$ at $p$ since the order of $\alpha$ is 3. So $\alpha$ preserves the three local branches of $C+H$ at $p$. This implies $E$ and hence $E+\tilde{H}$ is $\alpha$-fixed. By (1.11) $\alpha$ is trivial on $S$, a contradiction. Now we can assume that $p \in C$ is a node which can be resolved by at least two successive blowups. Then $p_{a}(C) \geq 2$ and $g(\tilde{C}) \leq 1$, where $\tilde{C}$ is the normalization of $C$. If $g(\tilde{C})=1$, by Lemma 3.3(ii), $\alpha$ is a translation of $\tilde{C}$, which is impossible since $\alpha$ preserves the local branches of $C$ at $p$. Now we assume $g(\tilde{C})=0$. If $F^{\prime}$ is reducible, let $D$ be an irreducible curve $D<F^{\prime}$ such that $D C>0$. Since $D \cap C$ is $\alpha$-invariant by Lemma 3.1 and there are exactly two $\alpha$-fixed points on $\tilde{C} \simeq \boldsymbol{P}^{1}$, we have $\#(D \cap C) \equiv 0(\bmod 3)$. This implies $p_{a}(C+D)>3$, a contradiction. Now we can assume $F^{\prime}=C$. If there is a point $q \in C_{\text {sing }} \backslash\{p\}$, then $q$ is $\alpha$-fixed and milt $q=2$ since $p_{a}(C)=3$, and hence there are at least four $\alpha$-fixed points on $\tilde{C}$. This implies $\alpha$ is trivial on $\tilde{C}$ and hence on $C$, a contradiction. So we can assume that $C \backslash\{p\}$ is smooth. Then $p$ is $\sigma$-fixed. If $\sigma$ preserves the local branches of $C$ at $p$, then $G$ also does. This implies $G$ is cyclic by (1.12), a contradiction. So we can assume $\sigma$ exchanges the local branches of $C$ at $p$. This implies there are two $\sigma$-fixed points on $C \backslash\{p\}$. Now by the same argument as in the last paragraph of Case 1, we get a contradiction. This completes the proof of (3.8.1).

\section{Proof of Proposition 2.7, the case $g=4,5$.}

Lemma 4.1. Let $f: S \rightarrow B, H, \Gamma_{i}, g$ and $G$ be as in (2.1). Assume that $g=4$ and $|G|=6$. Then $H$ is reduced and $\Gamma_{i}$ is nonsingular for every $i$.

ProOF. Let $F$ be a general fiber of $f$. Using the Hurwitz formula for $\pi: F \rightarrow F / G$, we get that $g(F / G)=1$ (note that $g(F / G) \geq 1$ by (1.15)) and $\pi$ has six ramification points. By (2.4.1), we have $\#(H \cap F) \geq 6$. This implies $H$ is reduced. Since $\#(H \cap F) \leq 2 g-2=6$, we have $R_{F}=H \cap F$. By (2.4.2), $\Gamma_{i}$ is nonsingular for every $i$.

LEMMA 4.2. Let $S$ be a minimal surface whose canonical map is composed with a pencil, and $f: S \rightarrow B$ the associated canonical fibration of genus $g$. Assume that $g=4$, and that the horizontal part $H$ of the fixed part of $\left|K_{S}\right|$ is reduced and each irreducible component of $H$ is nonsingular. Then $\chi\left(\mathcal{O}_{S}\right) \leq 188$.

Proof. Let the notation be as in (2.1). Under the assumption, we have

$$
K_{S} \equiv M+\sum_{i=1}^{t} \Gamma_{i}+V, \quad(t \leq 6)
$$


Let $g_{i}=g\left(\Gamma_{i}\right)$. From $K_{S} \Gamma_{i} \geq M \Gamma_{i}+\Gamma_{i}^{2}$ and the adjunction formula for $\Gamma_{i}$, we get

$$
K_{S} \Gamma_{i} \geq \frac{M \Gamma_{i}}{2}+g_{i}-1 .
$$

So

$$
\begin{aligned}
K_{S}^{2} & \geq K_{S} M+\sum K_{S} \Gamma_{i} \geq 6 d+\frac{\sum M \Gamma_{i}}{2}+\sum\left(g_{i}-1\right) \\
& =9 d+\sum\left(g_{i}-1\right) . \quad\left(\sum M \Gamma_{i}=M H=d F H=6 d\right)
\end{aligned}
$$

On the other hand, using the logarithmic Miyaoka-Yau inequality for $\left(S, \Gamma_{i}\right)(1.8)$, we have $K_{S}^{2} \leq 9 \chi\left(\mathcal{O}_{S}\right)+\left(g_{i}-1\right)-K_{S} \Gamma_{i} / 4$ for every $i$. Hence

$$
\begin{aligned}
K_{S}^{2} & \leq 9 \chi\left(\mathcal{O}_{S}\right)+\frac{\sum\left(g_{i}-1\right)}{t}-\frac{\sum K_{S} \Gamma_{i}}{4 t} \\
& \leq 9 \chi\left(\mathcal{O}_{S}\right)+\frac{3 \sum\left(g_{i}-1\right)}{4 t}-\frac{3 d}{4 t} \quad(\text { by }(1)) .
\end{aligned}
$$

Combining (2) and (3), we get

$$
3 d \leq(4 t-3) \sum\left(1-g_{i}\right)+36 t\left(\chi\left(\mathcal{O}_{S}\right)-d\right) .
$$

Note that $t \leq 6$, and $d=\chi\left(\mathcal{O}_{S}\right)$ if $g(B)=1$ and $d=\chi\left(\mathcal{O}_{S}\right)-2+q(S)$ if $g(B)=0$ (2.1.2). Hence we get $\chi\left(\mathcal{O}_{S}\right) \leq 188$.

Proof of Proposition 2.7, the CASE $g=4$. Let $f: S \rightarrow B$ be the canonical fibration associated with $\phi_{S}, F$ the general fiber of $f$, and $H$ the horizontal part of the fixed part of $\left|K_{S}\right|$. We have that $G$ induces the trivial action on $B$, and $G \hookrightarrow$ Aut $F$ by (2.2). By Lemma 2.5 , if $|G|>4$ then either $|G|=6$ or $G$ is a nonabelian group of order 8 or $12(|G| \neq 5$ by the Hurwitz formula).

First we suppose that $|G|=6$ or 12 . Then by Lemmas 2.5 and 4.1 , we have that $H$ is reduced and each irreducible component of $H$ is smooth. So by Lemma 4.2, $\chi\left(\mathcal{O}_{S}\right) \leq 188$, contradicting the assumption.

Second, we suppose that $G$ is a nonabelian group of order 8 . Then either $G \simeq D_{8}$ or $G \simeq Q_{8}$.

(i) The case $G \simeq D_{8}$ does not occur.

Otherwise, $D_{8} \hookrightarrow$ Aut $F$ for a general fiber $F$ of $f$. By (1.9), we have $4+2 g\left(F / D_{8}\right)=$ $g(F /\langle\alpha\rangle)+g\left(F /\left\langle\beta_{1}\right\rangle\right)+g\left(F /\left\langle\beta_{2}\right\rangle\right)$, where $\alpha, \beta_{i}$ are as in (1.9). But this is impossible since $g\left(F / D_{8}\right)=1, g(F /\langle\alpha\rangle)=1$, and $g\left(F /\left\langle\beta_{i}\right\rangle\right) \leq 2$ for every $i$ by the Hurwitz formula.

(ii) The case $G \simeq Q_{8}$ does not occur.

Otherwise, let $\sigma$ be a generator of $\operatorname{stab}(p)$ for some point $p \in H \cap F$. By the proof of Lemma 2.5, $\sigma$ is of order 4. Consider the commutative diagram

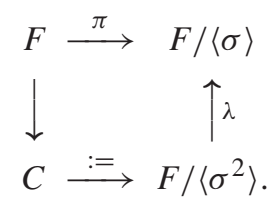


Since the ramification index of $\pi$ at $p \in F$ is $4, \lambda$ cannot be étale. This implies $g(C)=2$.

Since $Q_{8}$ has only one element of order $2,\left\langle\sigma^{2}\right\rangle$ is a normal subgroup of $Q_{8}$ and $\bar{G}:=$ $Q_{8} /\left\langle\sigma^{2}\right\rangle \simeq C_{2} \times C_{2}$. Using the Hurwitz formula for $C \rightarrow C / G \simeq F / Q_{8}$, (note that $g\left(F / Q_{8}\right)=1$,) by (1.13), we get $|\bar{G}| \leq 2$. This is a contradiction.

Proof of Proposition 2.7, The CASE $g=5$. Let $f: S \rightarrow B$ be the canonical fibration associated to $\phi_{S}$, and $F$ a general fiber of $f$. Let $M, H, V, \Gamma_{i}, n_{i}$ and $d$ be as in (2.1). Set $b=g(B)$. First we suppose that $n_{1}<g$. Since $n_{1} K_{S / B}+H+V$ is nef,

$$
\left(\left(n_{1}+1\right) K_{S}-M-n_{1}(2 b-2) F\right) H=\left(n_{1} K_{S / B}+H+V\right) H \geq 0 .
$$

So

$$
K_{S} H \geq \frac{(2 g-2)\left(d+n_{1}(2 b-2)\right)}{n_{1}+1} \geq \frac{(2 g-2)\left(d+n_{1}(2 b-2)\right)}{g} .
$$

On the other hand, using the Miyaoka-Yau inequality (cf. [Mi, Y]), we have

$$
9 \chi\left(\mathcal{O}_{S}\right) \geq K_{S}^{2}=K_{S}(M+H+V) \geq(2 g-2) d+K_{S} H .
$$

Combining these two inequalities, we get $\chi\left(\mathcal{O}_{S}\right) \leq 34$, which contradicts the assumption.

Now we can assume that $n_{1} \geq g$. Then $\Gamma_{1}$ is a section of $f$. This implies $\Gamma_{1}$ and hence the point $F \cap \Gamma_{1} \in F$ is $G$-fixed. By (1.12), $G$ is cyclic. Using the Hurwitz formula for $F \rightarrow F / G$, (note that $g(F / G) \geq 1(1.15)$ and by $(1.13)$ when $g(F / G)=1$ ) we get $G \simeq C_{5}$ and $\#(R \cap F)=2$ if $|G|>4$.

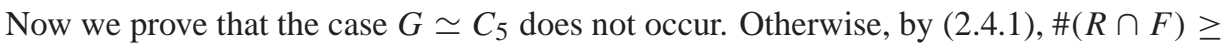
2. Since $\left(H-n_{1} \Gamma_{1}\right) F=8-n_{1} \leq 3$ and $|G|=5$, we must have $\#(R \cap F)=2$. So $H=n \Gamma_{1}+(8-n) \Gamma_{2}$ with $5 \leq n \leq 7$ and $\Gamma_{2} F=1$. Since $\Gamma_{1}+\Gamma_{2}$ is $G$-fixed, by (1.11), $\Gamma_{1} \Gamma_{2}=0$. From $K_{S} \Gamma_{1}=(M+H+V) \Gamma_{1} \geq d+n \Gamma_{1}^{2}$ and the adjunction formula for $\Gamma_{1}$, we get

Similarly, we have

$$
K_{S} \Gamma_{1} \geq \frac{d+n(2 b-2)}{n+1} .
$$

$$
K_{S} \Gamma_{2} \geq \frac{d+(8-n)(2 b-2)}{9-n} .
$$

Using the logarithmic Miyaoka-Yau inequality (1.8), we have

$$
\begin{aligned}
9 \chi\left(\mathcal{O}_{S}\right)+(b-1)-\frac{1}{4} K_{S}\left(\Gamma_{1}+\Gamma_{2}\right) & \geq K_{S}^{2}=K_{S}\left(M+n \Gamma_{1}+(8-n) \Gamma_{2}+V\right) \\
& \geq(2 g-2) d+n K_{S} \Gamma_{1}+(8-n) K_{S} \Gamma_{2} .
\end{aligned}
$$

Combining these inequalities, we get $\chi\left(\mathcal{O}_{S}\right) \leq 60$, which contradicts the assumption.

\section{REFERENCES}

[Ac] R. D. M. ACCOLA, Riemann surfaces with automorphism groups admitting partitions, Proc. Amer. Math. Soc. 21 (1969), 477-482.

[Be] A. BEAUVille, L'application canonique pour les surfaces de type qénéral, Invent. Math. 55 (1979), 121140. 
[BR] D. Burns And M. Rapoport, On the Torelli problem for Kählerian $K 3$ surfaces, Ann. Sci. École Norm. Sup. (4)8 (1975) 235-274.

[BPV] W. Barth, C. Peters and A. Van de Ven, Compact complex surfaces, Ergeb. Math. Grenzgeb. (3), Springer-Verlag, Berlin, 1984.

[Ca] J.-X. CAI, On Abelian automorphism groups of fiber surfaces of small genus, Math. Proc. Cambridge Philos. Soc. 130 (2001), 161-174.

[Fr] E. Freitag, Uber die Struktur der Funktionenkörper zu Hyperabelschen Gruppen I, J. Reine Angew. Math. 247 (1971), 97-117.

[Ha] R. Hartshorne, Algebraic Geometry, Graduate Texts in Mathematics 52, Springer-Verlag, New YorkHeidelberg, 1977.

[Mi] Y. MiYaokA, On the Chern numbers of surfaces of general type, Invent. Math. 42 (1977), $225-237$.

[Mu] D. Mumford, Algebraic Geometry I: Complex Projective Varieties, Springer-Verlag, 1976.

[Pe] C. A. M. PETERS, Holomorphic automorphisms of compact Kahler surfaces and their induced actions in cohomology, Invent. Math. 52 (1979), 143-148.

[Re] M. REID, Problems on pencils of small genus, Preprint (1990).

[Sa] F. SAKAI, Semi-stable curves on algebraic surfaces and logarithmic pluri-canonical maps, Math. Ann. 254 (1980), 89-120.

[Ue] K. UEnO, A remark on automorphisms of Enriques surfaces, J. Fac. Sci. Univ. Tokyo, Sect. I A Math. 23 (1976), 149-165.

[X1] G. XIAO, $\pi_{1}$ of elliptic and hyperelliptic surfaces, Internat. J. Math. 2 (1991), 599-615.

[X2] G. XIAO, L'irrégularité des surfaces de type général dont le système canonique est composé d'un pinceau, Compositio Math. (1985), 251-257.

[Y] S.-T. YAU, Calabi's conjecture and some new results in algebraic geometry, Proc. Nat. Acad. Sic. USA 74 (1977), 1789-1799.

LMAM

SChool of Mathematical Sciences and Institute of Mathematics

PEKING UNIVERSITY

BEIJING 100871

P. R. CHINA

E-mail address: cai@math.pku.edu.cn 